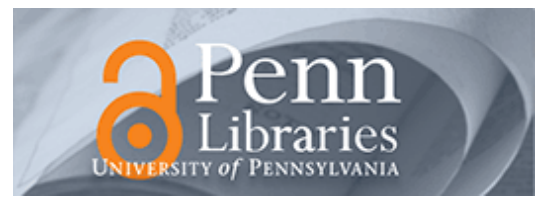

University of Pennsylvania ScholarlyCommons

$12-12-2006$

\title{
Comment on "Optical Response of Strongly Coupled Metal Nanoparticles in Dimer Arrays"
}

Vadim A. Markel

University of Pennsylvania, vmarkel@mail.med.upenn.edu

Follow this and additional works at: https://repository.upenn.edu/be_papers

Part of the Biomedical Engineering and Bioengineering Commons

\section{Recommended Citation}

Markel, V. A. (2006). Comment on "Optical Response of Strongly Coupled Metal Nanoparticles in Dimer Arrays". Retrieved from https://repository.upenn.edu/be_papers/179

Suggested Citation:

Markel, V.A. (2006). Comment on "Optical response of strongly coupled metal nanoparticles in dimer arrays" Physical Review B. 74, 216401.

(C) 2006 American Physical Society

http://dx.doi.org/10.1103/PhysRevB.74.216401

This paper is posted at ScholarlyCommons. https://repository.upenn.edu/be_papers/179

For more information, please contact repository@pobox.upenn.edu. 


\title{
Comment on "Optical Response of Strongly Coupled Metal Nanoparticles in Dimer Arrays"
}

\author{
Abstract \\ I have recalculated the extinction spectra of aggregates of two silver nanospheres shown in Figs. 2 and 3 \\ of the paper by J. J. Xiao, J. P. Huang, and K. W. Yu [Phys. Rev. B 71, 045404 (2005)]. I have used the \\ approximate method of images according to the formulas published in that reference and an exact \\ numerical technique. I have found that the three sets of data those I have obtained by the method of \\ images, the numerical results, and the results published in the reference in question do not coincide. In \\ this Comment, I discuss the reasons for these discrepancies and the general applicability of the method \\ of images to the quasistatic electromagnetic problem of two interacting nanospheres. \\ Disciplines \\ Biomedical Engineering and Bioengineering | Engineering

\section{Comments} \\ Suggested Citation: \\ Markel, V.A. (2006). Comment on "Optical response of strongly coupled metal nanoparticles in dimer \\ arrays" Physical Review B. 74, 216401. \\ (C) 2006 American Physical Society \\ http://dx.doi.org/10.1103/PhysRevB.74.216401
}




\title{
Comment on "Optical response of strongly coupled metal nanoparticles in dimer arrays"
}

\author{
Vadim A. Markel \\ Department of Radiology and Department of Bioengineering, University of Pennsylvania, Philadelphia, Pennsylvania 19104, USA
}

(Received 18 January 2006; revised manuscript received 1 June 2006; published 12 December 2006)

\begin{abstract}
I have recalculated the extinction spectra of aggregates of two silver nanospheres shown in Figs. 2 and 3 of the paper by J. J. Xiao, J. P. Huang, and K. W. Yu [Phys. Rev. B 71, 045404 (2005)]. I have used the approximate method of images according to the formulas published in that reference and an exact numerical technique. I have found that the three sets of data (those I have obtained by the method of images, the numerical results, and the results published in the reference in question) do not coincide. In this Comment, I discuss the reasons for these discrepancies and the general applicability of the method of images to the quasistatic electromagnetic problem of two interacting nanospheres.
\end{abstract}

DOI: 10.1103/PhysRevB.74.216401

PACS number(s): 82.70.-y, 41.20.Cv

The method of images (MOI) is a powerful tool for solving electrostatic problems. ${ }^{1}$ In particular, it has been used to calculate the electrostatic force between two charged spheres. $^{2}$ It was shown that the force can be well approximated by the Coulomb formula when the spheres are far apart. However, as the spheres approach each other, they cannot be effectively replaced by point charges and the Coulomb formula overestimates the actual force. The MOI was recently extended beyond the electrostatics. ${ }^{3-7}$ However, this generalization was the subject of some controversy. ${ }^{8,9}$ In particular, I have argued that the MOI, as used in the above references, is not a physically justifiable approximation and, therefore, cannot be used for calculating electromagnetic responses of interacting spheres at finite frequencies. ${ }^{8}$ I have further argued that the formulas derived in Refs. 3-7 do not provide accurate results even within electrostatics, when the MOI is, in principle, applicable. The authors of Ref. 9 argued that the MOI is an accurate approximation at sufficiently low frequencies, e.g., for $f<1 \mathrm{GHz}$. However, in Ref. 7, which is the subject of this Comment, the MOI is used for a dimer of silver nanospheres in the spectral range from 250 to $1500 \mathrm{~nm}$, i.e., at much larger electromagnetic frequencies. I have recalculated the data shown in Figs. 2 and 3 of Ref. 7 using the MOI as it is defined in Refs. 3-7. I have also calculated the relevant quantities using the exact method (e.g., see Ref. 10). I have found that the three sets of data (i.e., the data shown in Figs. 2 and 3 of Ref. 7, the data I have obtained according to the MOI using the same formulas as in Ref. 7, and the exact results) do not coincide. The deviations are significant. This and other points relevant to the applicability of the MOI are discussed in this Comment.

First, we specify the dielectric function of silver $\epsilon$, used in the calculations. This is given in Eq. (10) of Ref. 7 as a function of frequency $\omega$. Since the electromagnetic frequency is expressed in the units of energy in Figs. 2 and 3 of Ref. 7, we rewrite equivalently this equation as $\epsilon(E)=\epsilon_{h}$ $+E_{p}^{2} / E(E+i \Gamma)$, where $\epsilon_{h}=5.45$ is the bounded electron contribution to the dielectric function (assumed to be constant over the spectral region of interest), $E=\hbar \omega, E_{p}=9.68 \mathrm{eV}, \Gamma$ $=0.00181 \times E_{p}(1+2 \ell / d), \ell=52 \mathrm{~nm}$ is the electron free path, and $d$ is the sphere diameter. This expression, with the numerical values of parameters as given, provides a good approximation for the dielectric function of silver. The function $\epsilon(E)$ is plotted in Fig. 1 for different values of the sphere diameter $d$. An analogous graph is also shown in Fig. 1 of Ref. 7 for $d=10 \mathrm{~nm}$. While the real parts of the dielectric function are qualitatively similar in both figures, the imaginary parts (for $d=10 \mathrm{~nm}$ ) are very different. By comparison with other curves in Fig. 1, I infer that the dashed curve shown in Fig. 1 of Ref. 7 corresponds to the case $d \rightarrow \infty$ rather than to $d=10 \mathrm{~nm}$, as claimed.

The mathematical formalism of MOI (Refs. 3-7) is based on the Bergman-Milton spectral representation. ${ }^{11}$ Namely, the polarizability of each sphere in a two-sphere aggregate is written as

$$
\alpha=\frac{(d / 2)^{3}}{3} \sum_{n=1}^{\infty} \frac{F_{n}^{(L, T)}}{s+s_{n}^{(L, T)}},
$$

where $F_{n}^{(L, T)}$ is the oscillator strength of the $n$th polarization mode, $s_{n}^{(L, T)}$ are the generalized depolarization factors, $s$ $=\epsilon_{m} /\left(\epsilon-\epsilon_{m}\right)$ is the spectral parameter of the theory, with $\epsilon_{m}$ being the dielectric function of the transparent matrix in which the spheres are embedded, the upper index $L$ corresponds to polarization of the external field parallel to the axis of symmetry of two spheres, and the index $T$ corresponds to orthogonal polarization. We note that the factors $F_{n}^{(L, T)}$ of Eq. (1) differ from those of Refs. 3-7 by the overall factor of -3 and the spectral parameter $s$ by the factor of -1 . In particular, oscillator strengths that appear in Eq. (1) satisfy the sum rule $\sum_{n>0} F_{n}^{(L, T)}=1$. This corresponds to the more conventional no-

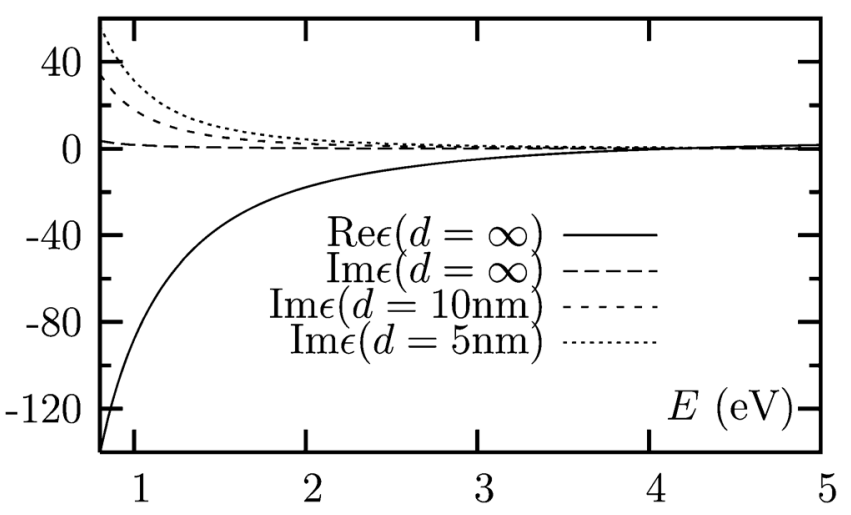

FIG. 1. Size-dependent dielectric function for different values of the sphere radius $d$ in the spectral range of interest. 

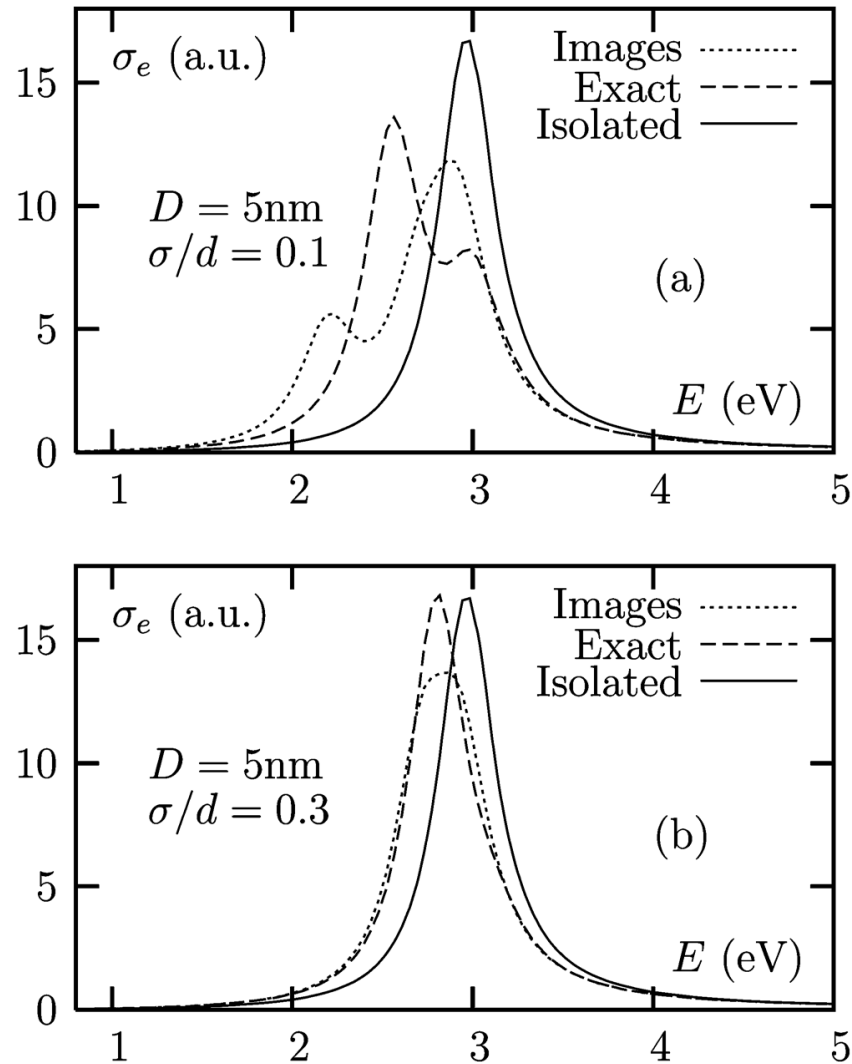

FIG. 2. Extinction spectra for a two-sphere aggregate in a transparent matrix with $\epsilon_{m}=1.61$ (Ref. 2) and $d=5 \mathrm{~nm}$. Polarization of the external field is parallel to the axis of symmetry. The exact results are compared to MOI and to the spectra for isolated (noninteracting) spheres.

tations adopted, for example, in Refs. 12-14. With the account of these two differences, Eq. (1) is completely equivalent to Eq. (5) of Ref. 7.

The spectral expansion (1) is quite general and is not the subject of a controversy. The values of $F_{n}^{(L, T)}$ and $s_{n}^{(L, T)}$ can be, in principle, found numerically. The potential advantage of the theoretical development of Refs. 3-7 is that it provides analytical expressions for these coefficients. These expressions are given in Eq. (7) of Ref. 7 (up to the numerical factor discussed above), or, in a form that allows direct substitution into Eq. (1), in Ref. 8. However, I have previously argued that (i) the values of these coefficients cannot be, in principle, found from MOI, even approximately, and (ii) the expressions suggested in Refs. 3-7 for these coefficients are inconsistent with the electrostatic $\operatorname{limit}^{8}$ (and, thus, are inapplicable even in the spectral range $f<1 \mathrm{GHz}$ ). Therefore, MOI does not provide a physically meaningful approximation. This is illustrated in Figs. 2 and 3 below.

In what follows, we consider only the results for the polarization of the external field being parallel to the axis of symmetry of two spheres [and omit the upper index " $(L)$ "], since the multipole interaction is strongest in this case. The surface-to-surface intersphere separation is denoted by $\sigma$ ( $\sigma=0$ for touching spheres). I have used the dielectric function defined above to calculate the extinction cross section of a bisphere aggregate for the same sets of parameters as in
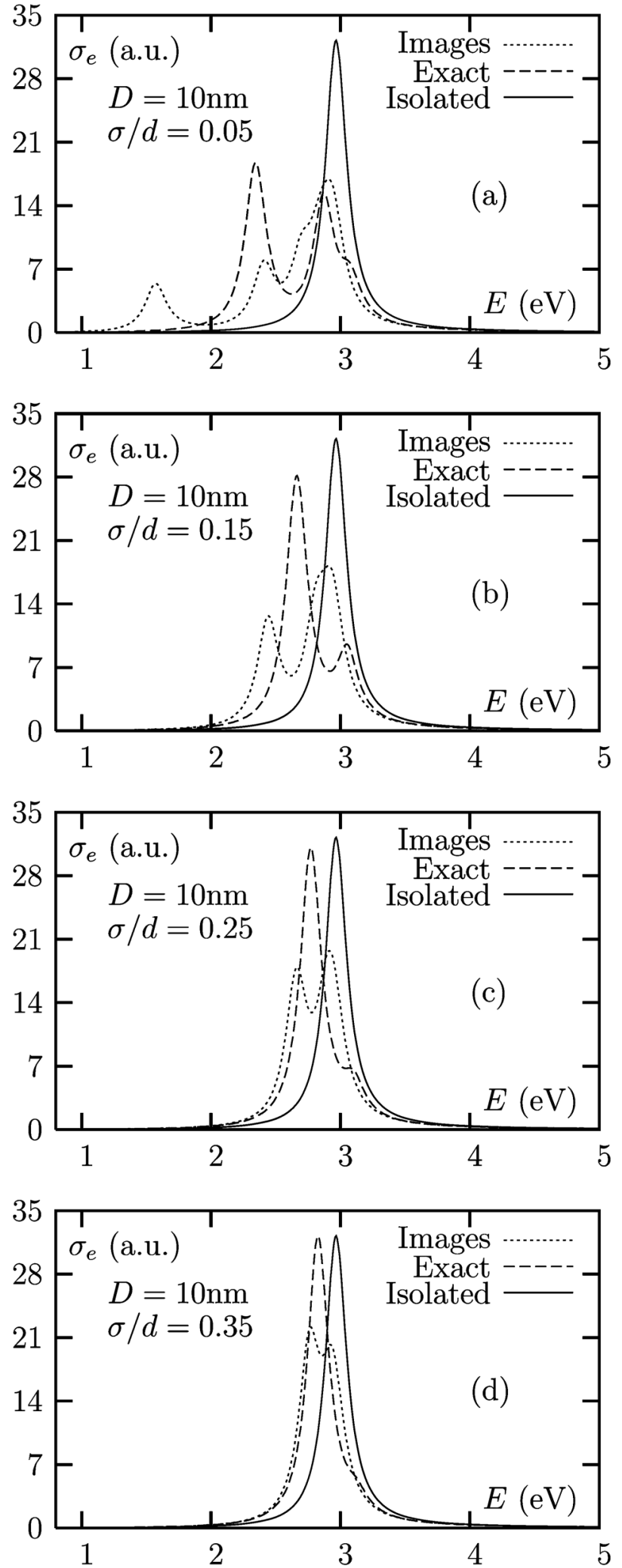

FIG. 3. Same as in Fig. 2, but for $d=10 \mathrm{~nm}$ and a different selection of the ratio $\sigma / d$.

Figs. 2 and 3 of Ref. 7. Namely, the dielectric constant of the matrix was $\epsilon_{m}=(1.61)^{2}$, the sphere diameters were chosen to be $d=5 \mathrm{~nm}$ (Fig. 2) and $d=10 \mathrm{~nm}$ (Fig. 3), and the ratio $\sigma / d$ was 0.1 and 0.3 (Fig. 2) and $0.05,0.15,0.25$, and 0.35 (Fig. 
3). The extinction in Ref. 7 was plotted in arbitrary units and not defined precisely. Therefore, I plot the quantity $(E / 3) \operatorname{Im} \Sigma_{n>0} F_{n} /\left(s+s_{n}\right)$, where $E$ is expressed in electron volts. This quantity differs from the actual extinction cross section only by an energy-independent overall factor, and I have found that it has approximately the same numerical values as the data shown in Figs. 2 and 3 of Ref. 7.

We first discuss Fig. 2, which is analogous to Fig. 2 of Ref. 7, except that data for orthogonal polarization are not shown. The exact spectra were calculated by the method of Ref. 10 with the maximum multipole order $L=400$ and convergence was verified by doubling this number. The MOI calculations were carried out by truncating summation in Eq. (1) at $n=400$, which is much more than is needed for convergence. First, it should be noted that the shape of the curves that I have obtained by the MOI are markedly different from those shown in Fig. 2 of Ref. 7. In particular, the second, low-energy peak in the extinction spectrum for $\sigma / d=0.1$ is significantly less pronounced in Ref. 7 than in my data. A noticeable deviation is also visible in the case $\sigma / d=0.3$. A possible cause of this discrepancy is discussed below. More importantly, the MOI curves in both cases differ from the exact result. The difference is very apparent at the smaller separation $(\sigma / d=0.1)$ and still visible at the relatively large separation $\sigma / d=0.3$.

The spectra in Fig. 2 are characterized by very strong relaxation because the ratio $2 \ell / d$ is in this case of the order of 20. Thus, the finite-size contribution to the relaxation constant is approximately 20 times larger than the respective constant in bulk. We then consider the case $d=10 \mathrm{~nm}$ when the relaxation is not as strong. The results are shown in Fig. 3, which corresponds to Fig. 3 of Ref. 7 with the exception that the results for orthogonal polarization are not shown. Again, there is a clearly visible difference between the MOI results obtained here and in Ref. 7. In all cases, the MOI spectra are very different from the exact spectra. This is especially apparent at the relatively small separation $\sigma / d$ $=0.05$ when the MOI predicts a spectral peak at $E \approx 1.5 \mathrm{eV}$, which is not present in the exact data. Even for the relatively large separation $\sigma / d=0.35$, the MOI produces a two-peak structure, while the exact spectrum has only one peak. [Note that in Fig. 3(d) of Ref. 7, the respective curve has also only one peak, but its maximum is about $10 \%$ smaller than the maximum of the spectrum in the noninteracting case. In the exact result, the maximum is approximately equal to that for the noninteracting case.]

The conclusion that can be drawn so far is that the MOI is inadequate for the spectral range and set of parameters used in Figs. 2 and 3 of Ref. 7. The inaccuracy of the MOI is especially evident at smaller intersphere separations and for larger sphere diameters.

We now discuss the possible cause of the discrepancy of the MOI calculations presented here and in Ref. 7. In Fig. 4 we plot the MOI curve for $\sigma / d=0.35$ and different values of $d$. The two-peak spectrum obtained at $d=10 \mathrm{~nm}$ is the same as the one shown in Fig. 3(d), while the single-peak spectrum obtained at $d=5 \mathrm{~nm}$ closely resembles the curve shown in Fig. 3(d) of Ref. 7. Thus, the possible cause of the discrepancy is that in Ref. 7 the actual value of the sphere diameter used in calculations was twice smaller than what is

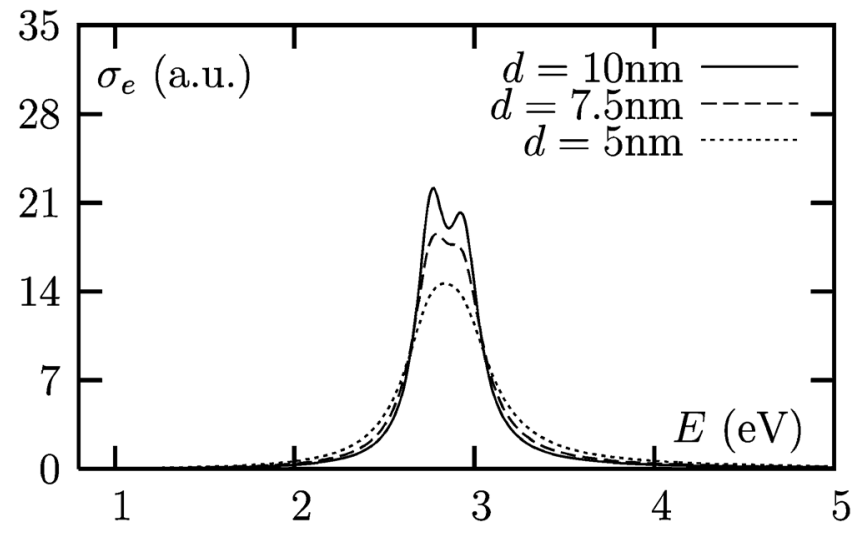

FIG. 4. The MOI result for the extinction spectrum of two spheres of different diameters $d$ and the ratio $\sigma / d=0.35$. Polarization of the external field is parallel to the axis of symmetry.

shown in the figure captions. That is, calculations in Fig. 2 of Ref. 7 were actually performed for $d=2.5 \mathrm{~nm}$ and in Fig. 3 of Ref. 7 for $d=5 \mathrm{~nm}$. Under these circumstances, the relaxation due to the finite-size effects is extremely strong and the spectral parameter $s$ has a large imaginary part, which effectively weakens the multipole interaction of the spheres.

The role of relaxation can be elucidated by considering the case when the complex spectral variable $s$ is sufficiently separated in the complex plane from all (purely real) numbers $s_{n}$. Then one can replace the denominator $s+s_{n}$ in Eq. (1) by $s$. (In the mean-field approximation, the denominators are replaced by $s+Q$, where $Q$ is the appropriate average of the interaction operator. ${ }^{15}$ ) The result is (taking into account the sum rule for $F_{n}$ 's) the polarizability of an isolated (noninteracting) sphere. We can further expand the result in powers of the small parameter $s_{n} / s$ and thus obtain corrections to the noninteracting result. Unlike the former, these corrections depend on the particular choice of $F_{n}$ and $s_{n}$. While the corrections are still small, the noninteracting result is modified only modestly and the resultant spectra may not display obvious anomalies and seem to be "qualitatively correct." An important point is that even if the corrections are small, they are not necessarily physically meaningful since there is an infinite number of ways to introduce arbitrary but small corrections. Still, while the corrections are relatively small, the exact and the MOI spectra may seem to coincide, at least qualitatively. However, any coincidences between the MOI and the exact spectra, such as the ones shown in Ref. 9, are due to weak electromagnetic interaction of nanospheres in which case the MOI spectra do not differ much from the spectra of noninteracting nanospheres.

One way in which the complex spectral variable $s$ can be removed far from the interval on the real axis, which contains the numbers $s_{n}$, is by introducing strong relaxation. In particular, this can be achieved by making the diameter $d$ in the formula $\Gamma=0.00181 \times E_{p}(1+2 \ell / d)$ for the Drude relaxation constant to be arbitrarily small. Then $s$ acquires a large imaginary part. This was the case in Ref. 7 where the electromagnetic interaction of nanospheres was suppressed by relaxation due to the finite-size effects. In addition, the diameter of nanospheres used in calculations of Ref. 7 appears to be one half of the value that is claimed in the figure captions. 
This resulted in relatively modest spectral changes (compared to the spectra of noninteracting spheres), which appear to be realistic. However, comparison with numerical results clearly demonstrates that even when the relaxation strength is overestimated, the MOI results are still inaccurate, and that this inaccuracy becomes very substantial for actual values of the relaxation constant (i.e., for $\Gamma$ corresponding to the values of $d$ shown in the figure captions).
${ }^{1}$ L. D. Landau and L. P. Lifshitz, Electrodynamics of Continuous Media (Pergamon Press, Oxford, 1984).

${ }^{2}$ J. A. Soules, Am. J. Phys. 58, 1195 (1990).

${ }^{3}$ K. W. Yu and T. K. Wan, Comput. Phys. Commun. 129, 177 (2000).

${ }^{4}$ J. P. Huang, K. W. Yu, and G. Q. Gu, Phys. Rev. E 65, 021401 (2002).

${ }^{5}$ L. Dong, J. P. Huang, and K. W. Yu, J. Appl. Phys. 95, 8321 (2004).

${ }^{6}$ J. P. Huang, M. Karttunen, K. W. Yu, L. Dong, and G. Q. Gu, Phys. Rev. E 69, 051402 (2004).

${ }^{7}$ J. J. Xiao, J. P. Huang, and K. W. Yu, Phys. Rev. B 71, 045404
(2005).

${ }^{8}$ V. A. Markel, Phys. Rev. E 72, 023401 (2005).

${ }^{9}$ J. P. Huang, K. W. Yu, G. Q. Gu, M. Karttunen, and L. Dong, Phys. Rev. E 72, 023402 (2005).

${ }^{10}$ V. A. Markel, V. N. Pustovit, S. V. Karpov, A. V. Obuschenko, V. S. Gerasimov, and I. L. Isaev, Phys. Rev. B 70, 054202 (2004).

${ }^{11}$ D. J. Bergman, Phys. Rep., Phys. Lett. 43, 377 (1978).

${ }^{12}$ R. Rojas and F. Claro, Phys. Rev. B 34, 3730 (1986).

${ }^{13}$ R. Fuchs and F. Claro, Phys. Rev. B 39, 3875 (1989).

${ }^{14}$ F. Claro and R. Fuchs, Phys. Rev. B 44, 4109 (1991).

${ }^{15}$ M. V. Berry and I. C. Percival, Opt. Acta 33, 577 (1986). 